\title{
Septic arthritis and osteomyelitis of the hip by salmonella montevideo in immunocompetent patient
}

\begin{abstract}
Salmonella usually produces enteric fever and gastroenteritis. However, the infection may spread through the blood stream and present as local infection at any site including the bone, joints, and central nervous system. The rate of osteomyelitis and septic arthritis secondary to Salmonella are rare conditions. Our case represents a patient with hip septic arthritis and osteomyelitis of the pelvis with unusual salmonella serotype Montevideo, group C1.
\end{abstract}

Volume 10 Issue I - 2020

\author{
Muhannad Antoun,' Kevin Pugh, ${ }^{2}$ Fadi Al- \\ Akhrass, ${ }^{3}$ Jeffery C Martin ${ }^{4}$ \\ 'University of Pikeville, Infectious Disease Department, USA \\ ${ }^{2}$ Pikeville Medical Center, Orthopedic surgery, USA \\ ${ }^{3}$ Pikeville Medical Center, Infectious Disease Department, USA \\ ${ }^{4}$ Pikeville Medical Center, Orthopedic Trauma, USA
}

Correspondence: Muhannad Antoun, University of Pikeville, Pikeville Medical Center, Infectious Disease Department, USA, Emailmuhannadantou@gmail.com

Received: January 22, 2020 | Published: January 30, 2020

\section{Introduction}

Salmonella is a non-lactose gram negative rod. It is commonly transmitted via fecal oral route. The most common manifestation is gastroenteritis. However, with no therapy, especially in patients with chronic diseases or in immunosuppressive state, that will put the patient in risk of bacteremia and extra intestinal infection.

\section{Case}

A 60 years old man presented to hospital due to right hip and right groin pain on ambulation with difficulty flexing the right thigh into his abdomen. The patient has history of hypothyroidism; hypertension; and diabetes mellitus. One month prior to his current presentation, he complained of gastroenteritis symptoms including; cramp like abdominal pain, nausea, vomiting, diarrhea, and fever, which subsided within a few days without treatment. His stool culture was positive for salmonella Group C1 Serotype Montevideo, sensitive to Ceftriaxone, Ampicillin, Levofloxacin and Septra.

Physical examination upon arrival was not significant other than motion restriction in the right hip. Vital signs with blood pressure of $115 / 77$, temperature $99.9 \mathrm{~F}$, heart rate of 113 per minute and respiratory rate 20per minute. The laboratory findings were White blood cells count 14.6K/UL, hemoglobin $11.1 \mathrm{gm} / \mathrm{dl}$, Platelet $297 \mathrm{~K} / \mathrm{UL}$, ESR $77 \mathrm{MM} / \mathrm{HR}$, CRP $30.3 \mathrm{mg} / \mathrm{dl}$, Creatinine $2.3 \mathrm{mg} / \mathrm{dl}$, ALT $58 \mathrm{U} / \mathrm{L}$, Hemoglobin A1C 10\%.

Radiographic study with MRI of the right hip without contrast showed right hip moderate-sized effusion with diffusely scattered soft tissue/intramuscular fluid and edema extending along the right iliac wing with diffuse marrow edema involving the right pelvis, right femoral head/neck region compatible with osteomyelitis and septic arthritis. (Figure 1)

CT guided needle aspiration of the right hip synovial fluid reveled $\sim 18 \mathrm{cc}$ of cloudy fluid. Analytic fluid of the fluid showed leukocyte count 79800 with $80 \%$ segment. That was sent to microbiology; Gram stain showed many WBC but with no organism seen. However, culture yielded non-lactose fermenting gram negative roads, which was confirmed as Salmonella Group C1, serotype Montevideo, sensitive to fluoroquinolone, ampicillin, Septra and Ceftriaxone. Blood culture was collected and sent to microbiology which turn to be positive for the same organism isolated from the right hip synovial fluid with the same sensitivities.

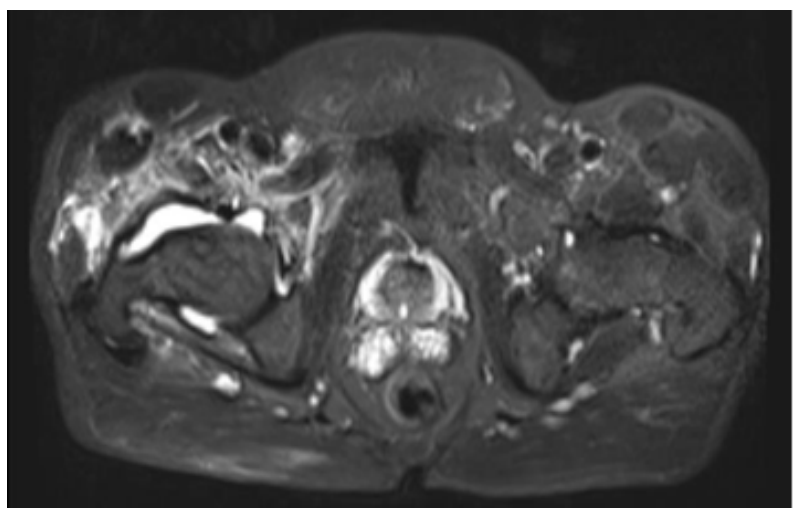

Figure I Right hip joint fluid collection and pelvic bone bone marrow edema.

He underwent surgery with irrigation and debridement of the right hip, along with debridement of the right iliac wing. Per operative note, there was purulent material that was evacuated from the capsule of the right hip and the right iliac wing.

Intraoperative specimen culture, again grew the same previous pathogen. Repeated blood culture prior to discharged was with no growth. The patient was treated with an 8 -week regimen: including 6 weeks of intravenous Ceftriaxone followed by 2 weeks of oral Levofloxacin, despite appropriate management. However, the patient continued to have severe pain in the right hip along with severe motion restriction. Two months later, MRI of the right hip with intravenous contrast was preformed and it showed worsening of the osteomyelitis and septic arthritis of the right pelvis, right femoral head/neck region. (Figure 2). 


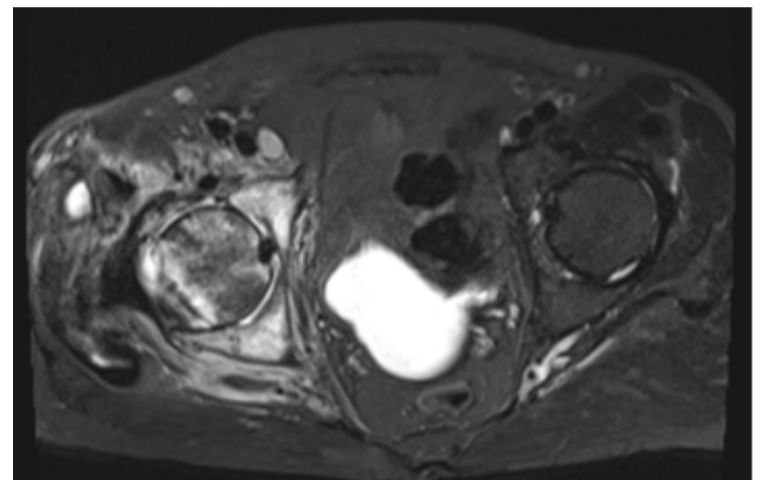

Figure 2 Bone marrow edema of the right hip head and adjacent pelvic bone.

The patient underwent right hip bipolar hemiarthroplasty with stage one antibiotic cement spacer, then a revision of right total hip arthroplasty and revision of femoral and acetabular as a stage two surgery. During the revision surgery, 4 frozen section synovial tissue specimens were obtained and sent to pathology which all showed zero neutrophils per high-power field. His inflammatory markers prior to surgery were ESR $18 \mathrm{MM} / \mathrm{HR}$ and CRP $1.2 \mathrm{mg} / \mathrm{dl}$.

\section{Discussion}

Salmonellae are facultative anaerobic gram negative bacilli. Transmitted with fecal oral route. It can cause multiple types of infection including gastroenteritis, enteric fever, bacteremia, endovascular infections, osteomyelitis, septic arthritis and abscesses. Other route of transmission is animal contact with reptiles and amphibians. Septic arthritis and gastroenteritis due to Salmonella species were reported in children with sickle cell disease after exposure to reptiles or amphibians. ${ }^{1}$ Cases of reptile-associated salmonellosis in children $\leq 5$ years of age occurring in Michigan between January 2001 and June $2003 .^{2}$

It is characterized based on three surface antigens; somatic $\mathrm{O}$ lipopolysaccharide antigens, flagellar $\mathrm{H}$ antigens, and Vi capsular polysaccharide. ${ }^{3}$ Specific serotypes were defined as a result of complex antigen variability. The result was identifying more than 2,500 serotypes for Salmonella; but less than 100 serotypes account for most human infections. ${ }^{4}$ The $\mathrm{O}$-antigen comprises many repeats of an oligosaccharide. This repeated unit varies among strains and in Salmonella enterica there are about 60 different forms. ${ }^{5}$ It is widely used by clinical laboratories to divide Salmonella into serogroups A, $\mathrm{B}, \mathrm{C} 1, \mathrm{C} 2, \mathrm{D}$, and $\mathrm{E}$.

Salmonella divided into typhoidal and nontyphoidal types. However, the nontyphoidal serotypes are more common to cause bacteremia and focal infection; osteomyelitis and septic arthritis. Factors contributing to these complications include; immunosuppressive therapy, TNF blockade (etanercept or infliximab), sickle-cell disease, alcoholism and HIV infection. Other serotype can be more invasive like Salmonella enterica.

Salmonella enteritidis and Salmonella typhimurium were the most common serotype causing bone infections, whereas members of the $\mathrm{C} 1$ serogroup including (Choleraesuis, Virchow, Infantis and Montevideo) were the most common cause of septic arthritis with representation up to $67 \%{ }^{6}$
Salmonella -Montevideo infection was associated with handling live poultry, reptiles and imported spices. ${ }^{7,8}$ Different sites of infection other than the intestine like myocardium and pulmonary tissue were reported caused by this specie. ${ }^{9,10}$ A case of septic arthritis of the knee was reported after 3 months of enteritis caused by S. Montevideo in a patient with longterm use of corticosteroids. ${ }^{11}$

\section{Conclusion}

Uncontrolled diabetes mellitus patients are at high risk for complicated salmonella gastroenteritis with disseminated infection. Pyogenic joint infection and osteomyelitis are one of the extraintestinal site infections. Management requires combination of surgical approach, as well as long course of antibiotic therapy.

\section{Acknowledgments}

None.

\section{Conflicts of interest}

The authors declare that they have no conflicts of interest regarding this article.

\section{Funding}

None.

\section{References}

1. Boguniewicz J, Rubiano-Landinez A, Lamb G, et al. Comparison of musculoskeletal infections due to non-typhoidal Salmonella species and Staphylococcus aureus in immunocompetent children. Pediatr Infect Dis J. 2019;38(10):1020-1024.

2. Wells EV, Boulton M, Hall W, et al. Reptile-associated salmonellosis in preschool-aged children in Michigan, January 2001-June 2003. Clin Infect Dis. 2004;39(5):687-691.

3. Kauffman F. The diagnosis of Salmonella types. Charles C Thomas, editor. Springfield Ill. 1950.

4. SandraI.Berríos T, Craig AU, Dale W, et al. Centers for Disease Control and Prevention. Jama Surgery. 2017;152(8)785-791.

5. Journal of Bacteriology. American Society for Microbiology. 1993.

6. Mary M Eichorn A. The Journal of Infectious Diseases: Yesteryear and Today. The journal of infectious diseases. 1978;138(6):709-711.

7. Fonseca RJ, Dubey LM, S montevedio sepsis from pet snake. Paeriatr infec dis J. 1994;13(6):550.

8. Morbidity and Mortality Weekly Report MMWR. 1997;46(11):237-239.

9. O'connor K. Acute myocarditis precipitated by Salmonella Montevideo infection: a case report. Ir med J. 2000;93(1):21-22.

10. Montane de la roque P, Ascensio R, Giauffret F, et al. Salmonella Montevideo hypoxemic pleuropneumonia. Rev Med Interne. 200, 21:203-204.

11. Katsoulis E, Pallett A, Bowyer GW. Septic arthritis of the knee by Salmonella montevideo. Ann R Coll Surg Engl. 2004;86(4):272-274. 